Relations industrielles

Industrial Relations

\title{
Les tribunaux du travail
}

\section{Propos préliminaires}

\section{Gérard Dion}

Volume 16, numéro 3, juillet 1961

URI : https://id.erudit.org/iderudit/1021774ar

DOI : https://doi.org/10.7202/1021774ar

Aller au sommaire du numéro

\section{Éditeur(s)}

Département des relations industrielles de l’Université Laval

ISSN

0034-379X (imprimé)

1703-8138 (numérique)

Découvrir la revue

Citer ce document

Dion, G. (1961). Les tribunaux du travail : propos préliminaires. Relations industrielles / Industrial Relations, 16(3), 348-350.

https://doi.org/10.7202/1021774ar
Résumé de l'article

Les 10 et 11 avril, le Département des relations industrielles de Laval tenait son 16ème Congrès des relations industrielles. Le texte des travaux présentés sera bientôt publié dans un volume. Nous donnons ici un résumé des communications.
Tous droits réservés (C Département des relations industrielles de l’Université Laval, 1961
Ce document est protégé par la loi sur le droit d'auteur. L’utilisation des services d'Érudit (y compris la reproduction) est assujettie à sa politique d'utilisation que vous pouvez consulter en ligne.

https://apropos.erudit.org/fr/usagers/politique-dutilisation/ 
Le président et l'arbitre syndical sont donc d'opinion que, dans les circonstances, le congédiement de Dame X n'était pas justifié.

SENTENCE: Le tribunal d'arbitrage, à la majorité, l'arbitre patronal étant dissident, enjoint l'employeur de réintégrer madame $\mathrm{X}$ dans son emploi sans aucune perte de classement ni de séniorité, tout en la changeant de département de façon qu'elle n'ait pas à être sous les ordres de l'hospitalière du service central dont il a été question dans la preuve, et de lui accorder une indemnité équivalente à tout le salaire qu’elle a perd̉u à compter du 19 décembre 1960 jusqu’à la date de sa réintégration, à raison de $\$ 34.00$ par semaine jusqu'au 10 avril 1961 , et de $\$ 35.00$ à compter de celte date, moins la perte d'une semaine de salaire, $\$ 34.00$.

Vu les dispositions de l'article 172 (8) des Règlements sur l'assurance chômage et la circulaire du Commissaire en date du 30 août 1960, l'employée devra rembourser ses prestations d'assurance-chômage. Il ne peut donc y avoir lieu à compensation en faveur de l'employeur pour les prestations qu'elle a retirées pendant qu'elle ètait sans travail.

\section{INFORMATIONS}

\section{LES TRIBUNAUX DU TRAVAIL}

Les 10 et 11 avril, le Département des relations industrielles de Laval tenait son 16ème Congrès des relations industrielles. Le texte des travaux présentés sera bientôt publié dans un volume. Nous donnons ici un résumé des communications.

\section{Propos préliminaires}

\section{Gérand Dion}

Le Département des relations industrielles de l'Université Laval a inscrit au programme de son 16ème congrès annuel l'étude du problème des tribunaux $d u$ travail.

Cette question est loin d'ètre nouvelle chez nous. Elle possède cependant une certaine originalité puisque l'on pourrait écrire une assez longue histoire de ses rebondissements périodiques et de son étouffement successif sous l'action de forces diverses et mystérieuses.

La création des tribunaux du travail est une réclamation que l'on trouve encore contenue dans le cahier des revendications présentées en 1960 par la Confédération des syndicats nationaux au gouvernement de la province de Québec. C'est en 1935 - donc, il y a déjà 26 ans - que cette centrale syndicale, à son Congrès de Hull, adoptait pour la première fois une résolution dans ce sens. Et depuis lors, à chaque année, avec une patience et une constance qui frôlent la routine, elle en fait part aux gouvernements qui se succèdent. 
En 1937 - il y a 24 ans - une commission d'étude et de revision de la loi relative à l'extension des conventions collectives de travail avait été chargée par le gouvernement de la province de Québec d'enquêter sur la législation du travail et de rédiger une nouvelle loi. Cette commission, formée de Mes Guy Hudon, MarieLouis Beaulieu, Robert Lafleur et T.-P. Slattery, après avoir mentionné que presque toutes les délégations entendues ont insisté sur la nécessité des tribunaux du travail, en a recommandé l'institution.

En 1941, à la demande du Ministère du travail, Me Marie-Louis Beaulieu, qui était alors et qui est encore professeur de législation du travail à la Faculté des sciences sociales et à la Faculté de droit de l'Université Laval, préparait un mémoire dans lequel il disait:

« Il est difficile de s'attendre à ce que les juges des juridictions ordinaires possèdent les connaissances techniques, les connaissances de spécialités qui souvent nécessitent l'application de lois ouvrières. L'on doit dire qu'une magistrature du travail peut être instituée avec avantage dans un pays industrialisé et que parfois, la chose devient nécessaire. » travail.

Depuis vingt ans, ce mémoire repose en paix dans les archives du ministère du

Le Barreau de la province de Québec, lors de son premier congrès, en 1943, a étudié l'établissement de tribunaux du travail et en a approuvé le principe sous certaines conditions. Me Albert Leblanc y déclarait:

«Si nous tenons compte de l'état actuel des esprits, des demandes des ouvriers et des conditions de travail ne devons-nous pas nous demander si le temps n'est pas venu de considérer l'établissement de ces tribunaux spéciaux dits tribunaux du travail? Ils existent dans la plupart des pays... Nous sommes convaincus que tôt ou tard, nous aurons des tribunaux du travail...»

Cette observation date de 18 ans...! Tout comme la recommendation contenue dans le Rapport Prévost soumis par la Commission chargée de faire enquête sur certaines difficultés survenues aux usines de Price Brothers et de St. John Paper dont une partie seulement a été intégrée dans la loi des relations ouvrières, en 1944.

Le Département des relations industrielles de Laval n'a pas attendu jusqu'à ce jour pour aborder la question des tribunaux du travail.

Déjà, à son premier congrès, en 1946, un de nos professeurs, Me Louis-Philippe Pigeon, exprimait son point de vue. Plus tard, en 1954, nous avons consacré tout un congrès au Règlement des CONFLITS DE DROIT. Les communications de Me Théodore Lespérance, aujourd'hui juge et président de la Commission des relations ouvrières et de Me Emile Colas portaient spécifiquement sur cette question. Enfin, en 1949, un de nos brillants élèves, Me Benoît Yaccarini, faisait sa thèse de maîtrise au Département des relations industrielles sur les tribunaux du travail. Cette thèse a été, par la suite, publiée dans la Revue du Barreau et dans une brochure de la Faculté des sciences sociales.

Qu'est-ce donc qui a incité le Département des relations industrielles à revenir à la charge et à consacrer tous les travaux d'un congrès à l'étude des tribunaux du travail? 
La première raison, c'est qu'il s'agit d'un problème sérieux, important, qui n'a jamais été résolu d'une façon systématique. On ne peut empêcher l'évolution sociale. Toute société se doit de posséder les mécanismes nécessaires à la sauvegarde de l'ordre public et des droits de ses ressortissants. Le problème du règlement des conflits de clroit en matière de relations du travail est crucial. Non seulement les droits des personnes et des institutions économico-sociales sont en jeu, mais souvent la paix et l'ordre de toute la société. Les conflits de droit en relations du travail, qu'ils soient individuels ou collectifs, existent. On ne peut les éviter. Il faut qu'ils soient réglés. Il est vrai que les tribunaux communs s'en occupent, que des commissions administratives sont graduellement amenées à exercer des fonctions judiciaires. Mais, est-ce suffisant? Est-ce satisfaisant? Est-ce que l'on doit continuer à se contenter de remèdes partiels sans tenir compte de solutions d'ensemble? Peut-ètre que oui, peut-ètre que non. Il importe pour le bien de tous que la lumière soit faite, que l'on sache où l'on va.

La seconde raison qui nous a poussé à étudier les tribunaux du travail, c'est que lcur création se trouve à être un des articles du programme électoral du parti qui vient de prendre le pouvoir (art. 30). Jusqu'à quel point faut-il accorder du sérieux à un programme électoral? L'histoire nous a appris à garder beaucoup de réserve là-dessus et nous laissons à chacun le soin de porter son propre jugement. Mais il est bon de se préparer à toute éventualité. Ce n'est que simple prudence. Dans ce cas-ci, c'est un motif supplémentaire pour s'empresser de déblayer le terrain, de faire une étude objective avant que la passion, les intérêts, le parti-pris n'entrent en scène.

Le Département des relations industrielles de Laval n'est pas à la solde de qui que ce soit: gouvernement, syndicats ouvriers ou employeurs. Il est au service du public. Son rôle n'est pas d'apporter des solutions toutes faites, de dicter des lignes de conduite, mais bien de poser les problèmes, de stimuler la pensée, d'établir toutes les hypothèses, laissant ensuite aux intéressés le soin de prendre les décisions.

Le congrès des relations industrielles de Laval réunit tous ceux qui dans cette province s'occupent des relations du travail tant sur le plan académique que sur le plan de l'action. Il est une occasion pour tous de se rencontrer sur un terrain neutre et de discuter leurs problèmes dans des perspectives d'objectivité. C'est ce que nous voulons faire avec celui des tribunaux du travail.

\section{Nature et raisons d'être des tribunaux du travail}

\section{EMILE Gosselin}

L'accélération du rythme de l'industrialisation a vu s'accroître la fréquence et l'étendue de l'intervention gouvernementale. Il en est résulté une abondante législation sociale accompagnée d'une forte délégation de pouvoirs réglementaires et quasi-judiciaires à de nombreux organismes publics ou quasi-publics. L'Etat a non seulement obligé les individus et les groupes à de nouvelles façons d'agir, mais en élaborant un droit social différent de l'ordre juridique antérieur, il a consacré des droits nouveaux et, dans une certaine mesure, reformulé une nouvelle échelle de valeurs juridiques. 